\title{
A Reconfigurable Passive UHF Reader Loop Antenna for Near-Field and Far-Field RFID Applications
}

\author{
A. L. Borja, A. Belenguer, J. Cascon and J. R. Kelly
}

\begin{abstract}
This letter presents a passive UHF printed loop antenna with left-handed loading. The RFID reader antenna can work at near- and far-field operation within the European UHF $(865-868 \mathrm{MHz})$ band. The operation frequency and radiation properties can be modified by employing varactor diodes. Also, the antenna can work in the near-field region having a flat magnetic distribution in the interrogation zone. A comparative study with other passive RFID reader conventional antennas shows that the left-handed loop achieves stronger near $H$ field with good impedance matching. Design details, simulated results and a fabricated prototype are presented. The concept significantly extends the design degrees of freedom for RFID antennas.
\end{abstract}

Index Terms-Metamaterials, radiofrequency identification (RFID), left-handed loading, loop antenna.

\section{INTRODUCTION}

$\mathbf{I}$ $\mathrm{N}$ recent years, significant research has been conducted in the area of ultrahigh frequency (UHF) near/far-field radio frequency identification (RFID). RFID technology has gained popularity for a variety of applications such as contactless payment, metro tickets, keyless vehicle ignition systems, supply chain management, security or tracking items [1], [2]. Nearor far-field operation is used reliant on the tag and application characteristics. Near-field operation can be useful for objects surrounded by metals and/or liquids in their vicinity and farfield interaction is commonly used to achieve long read range. For this reason, there is significant research interest in developing antennas that can be used in these systems [3] - [7]. Lefthanded metamaterials operating at microwaves frequencies could also be applied in RFID applications. Generally, lefthanded antennas are created by assembling a periodic structure composed of elements which are small in size compared to the wavelength of the propagating electromagnetic wave. Consequently, the properties of these artificial materials no longer depend exclusively on the properties of the constitutive elements. Instead they depend on the properties of the whole macrostructure thus formed. The resulting material can be engineered to exhibit novel electromagnetic properties, and

Manuscript received. This work has been supported by Ministerio de Ciencia e Innovacion, Spanish Government, under Research Project TEC201021520-C04, and by the Autonomous Government of Castilla-La Mancha under Research Project PPII10-0027-1277.

Alejandro L. Borja, Angel Belenguer and Joaquin Cascon are with Universidad de Castilla-La Mancha, Departamento de Ingeniera Elctrica, Electrnica, Automtica y Comunicaciones Campus Universitario, 16071, Cuenca, Spain. e-mail: (Alejandro.Lucas@uclm.es).

James R. Kelly is with the University of Birmingham, School of Electronic, Electrical and Computer Engineering, Edgbaston, Birmingham B15 2TT, UK. to achieve behaviors that are not found with conventional materials.

In this paper, we present a novel loop antenna incorporating a ladder network with left-handed loading. The antenna exhibits the unique feature of antiparallel phase and group velocity along with zero propagation constant at a certain frequency [8] - [11]. In particular, the propagation constant of an artificial transmission line can reach zero values at frequencies where the phase advance and delay offsets cancel. Making use of this phenomenon, electrically small antennas with several operating modes [12] - [14] can be developed. More specifically, the loop antenna has been designed to exhibit zeroth order mode operation $(n=0)$ at a frequency of $868 \mathrm{MHz}$. The main advantage of zeroth order mode operation includes the ability to obtain an uniform current distribution which is independent of the loop length, reconfigurable performances and strong magnetic near-field distributions.

The paper is organized as follows. Section 2 discusses the antenna design of the passive UHF reader left-handed loop antenna. Sections 3 and 4 present the main properties of the structure in the near- and far-field. The performance is also compared with that of other conventional right-handed antennas. Finally, the main conclusions of the study and future possible applications are outlined in section 5 .

\section{LOOP ANTENNA DESIGN}

Fig. 1 (a) shows the loop antenna. The antenna is loaded by a left-handed ladder network composed of 4 unit cells. Moreover, Fig. 1 (b) shows a prototype which was fabricated using a mechanical milling machine (LPKF Protomat S100). The FR4 has a thickness of $0.6 \mathrm{~mm}$, a permittivity of $\epsilon_{r}=$ 4.4 and loss tangent of $\operatorname{tg} \delta=0.02$. Metal utilized is copper with a thickness of $35 \mu \mathrm{m}$, and a conductivity of $\sigma=5.8 \mathrm{x}$ $10^{7} \mathrm{~S} / \mathrm{m}$. The unit cell consist of lumped capacitors (denoted $C_{L}$ ) in the series arm, and lumped inductors (denoted $L_{L}$ ), in the shunt arm. The capacitors $C_{L}$ are introduced into the arm of the ladder network which also incorporates the feeding point. This leads to a difference in the amplitudes of currents $I_{1}$ and $I_{2}$. For this reason these out-of-phase currents do not completely cancel in the far field, and as a result they radiate. Thus, the proposed antenna allows size reduction whilst maintaining good impedance matching by the adjustment of $L_{L}$ and $C_{L}$. 

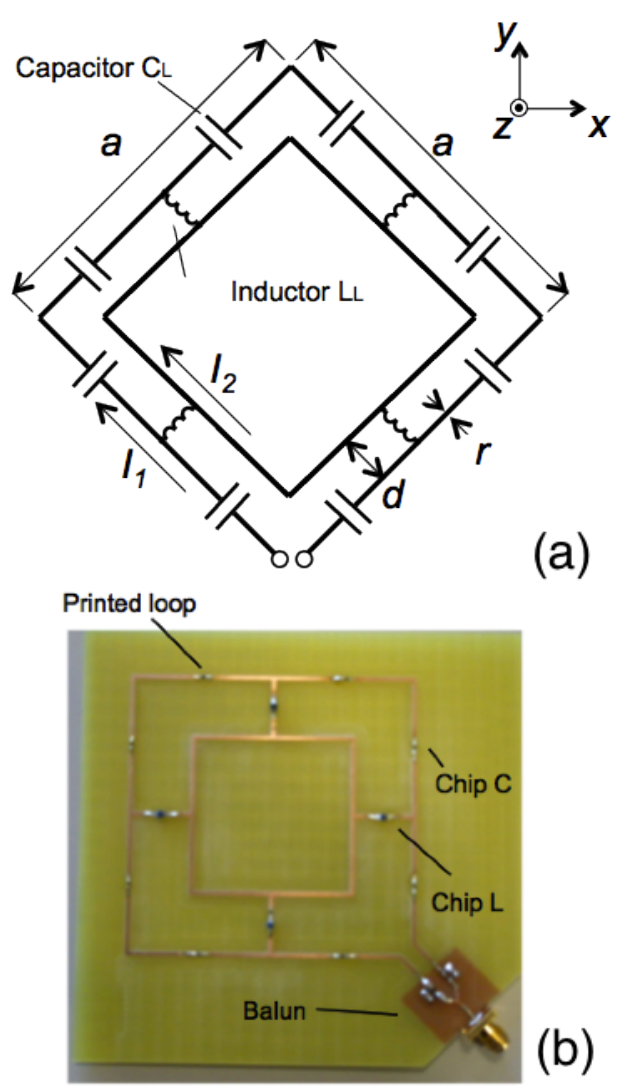

(b)

Fig. 1. (a) Model and (b) fabricated prototype of the loop antenna with left-handed transmission line. Loop parameters are $C_{L}=0.79 \mathrm{pF}, L_{L}=200$ $\mathrm{nH}, a=72.3, d=14.1$ and $r=0.7$. The fabricated antenna is fed through a balun that consists of a high pass filter, a low pass filter and a T-junction. Both filters have the fifth-order butterworth function. The balun has impedance of $50 \Omega$ for the balanced port and $50 \Omega$ for the unbalanced port. The balun introduces a small extra loss of about $0.4 \mathrm{~dB}$. A equivalent series resistance (ESR) of $577 \mathrm{~m} \Omega$ and $660 \mathrm{~m} \Omega$ for the capacitors and inductors, respectively, are considered. Units are in $\mathrm{mm}$.

\section{FAR-FIELD UHF RFID APPLICATION}

Fig. 2 shows the simulated and measured return loss performance for the proposed antenna. It also shows the simulated surface current distribution. Numerical simulations were performed using the commercial simulator CST 2011.

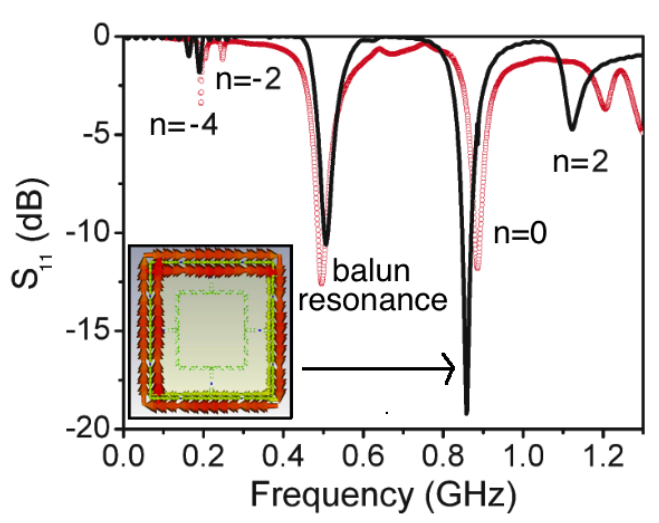

Fig. 2. Simulated (black solid line) and measured (red open symbol) return loss for the 4 unit cell left-handed loop antenna. The inset shows the current distribution at the $n=0$ resonance.
Four different operation modes can be observed. These can be understood by considering conventional resonance numbering, where the first and second resonance correspond to the left-handed region (negative resonances), the third resonance is attributed to the $n=0$ mode and the last resonance is related to the right-handed region (positive resonances). The left-handed resonances appears at lower frequencies than the right-handed ones. The $n=-2$ and $n=-4$ modes are observed at around 175 $\mathrm{MHz}$ and $200 \mathrm{MHz}$ according to simulation, and at $200 \mathrm{MHz}$ and $255 \mathrm{MHz}$ according to measurement. Similarly, the $\mathrm{n}=2$ mode is found at $1110 \mathrm{MHz}$ according to simulation and 1200 $\mathrm{MHz}$ according to measurement. An additional resonance is observed in the $S_{11}$ parameter between the left- and righthanded resonances. This occurs at $868 \mathrm{MHz}$ according to simulation and at $886 \mathrm{MHz}$ according to measurement. It correspond to the $\mathrm{n}=0$ resonance where the phase constant $\beta$ is close to zero. It is important to note that the $n=0$ mode operation can only be obtained using certain lumped element $L_{L}$ and $C_{L}$ values. The lower reflection coefficient $S_{11}$ at 500 $\mathrm{MHz}$ correspond to a balun resonance. Agreement between simulation and measurement is good, however some discrepancy can be observed. This is attributed to the tolerances in the lumped elements and the substrate material. In addition, Fig. 2 depicts the current distribution associated with the $n=0$ mode (868 MHz). Inspection of the inset reveals higher current flow around the outer loop $\left(I_{1}\right)$ in comparison with the inner loop $\left(I_{2}\right)$. The two currents are unidirectional. Moreover, even if the total perimeter of the loop is comparable to the operating wavelength, both currents $I_{1}$ and $I_{2}$ are uniformly distributed because the phase constant $\beta$ is near zero.

The left-handed loop antenna is now analyzed with the capacitors that load the outer loop of the antenna replaced by varactor diodes. The resonant frequency of the $n=0$ mode is controlled by the loading values. Consequently, a tunable passive UHF reader loop antenna with a reconfigurable response for RFID applications can be designed. The inductors are fixed to $L_{L}=200 \mathrm{nH}$, but the value of the capacitors can be varied if implemented as varactor diodes. Fig. 3 shows the reflection coefficient of the proposed antenna for different values of the variable capacitors.

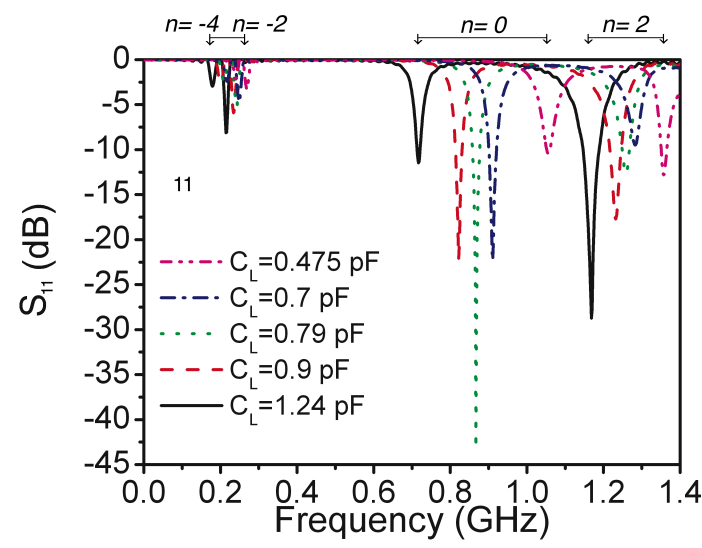

Fig. 3. Return loss of the proposed tunable frequency passive UHF reader loop antenna. 
In between the left- and right-handed regions, we can achieve the zero $(n=0)$ mode with a wide working frequency range. More specifically, the tuning range for the $\mathrm{n}=0$ mode goes from $717 \mathrm{MHz}\left(C_{L}=1.24 \mathrm{pF}\right)$ to $1056 \mathrm{MHz}\left(C_{L}=\right.$ $0.475 \mathrm{pF})$. The antenna is well matched $\left(S_{11} \leq-10 \mathrm{~dB}\right)$ for all the values. This means a tuning bandwidth of 39 $\%$. Moreover, different parameters at each shifted resonance for the $\mathrm{n}=0$ mode are presented in Table I. The directivity refers to the maximum value of directivity, usually obtained in the $\mathrm{x}-\mathrm{y}$ plane for this configuration. The total efficiency $\left(E f f_{t}\right)$ is defined as the ratio of radiated to stimulated power of the antenna. Compared to the input power, the stimulated power considers reflections occurring at the feed location. The reflection efficiency $\left(E f f_{r}\right)$ is defined as the ratio of input to stimulated power. Note that the input power is the accepted power i.e. reflected power and power flowing out any port of the simulated structure are not included.

TABLE I

STUDY OF THE FREQUENCY TUNABLE UHF LOOP ANTENNA WORKING AT THE N=0 MODE.

\begin{tabular}{|c|c|c|c|c|c|c|}
\hline $\begin{array}{c}\text { Res. } \\
(M H z)\end{array}$ & $\begin{array}{c}C_{L} \\
(p F)\end{array}$ & $\begin{array}{c}L_{L} \\
(n H)\end{array}$ & $\begin{array}{c}\text { Dir. } \\
(d B i)\end{array}$ & $\begin{array}{c}E f f_{r} \\
(\%)\end{array}$ & $\begin{array}{c}E f f_{t} \\
(\%)\end{array}$ & $\begin{array}{c}\text { Imp. } \\
(\Omega)\end{array}$ \\
\hline \hline 1056 & 0.475 & 200 & 2.14 & 87.6 & 80 & 83.9 \\
\hline 910 & 0.7 & 200 & 2.2 & 83.8 & 83.4 & 55.9 \\
\hline 868 & 0.79 & 200 & 2.14 & 82 & 81.9 & 50 \\
\hline 822 & 0.9 & 200 & 2.12 & 79.8 & 78.9 & 40.6 \\
\hline 717 & 1.24 & 200 & 2.04 & 72.9 & 65.9 & 26.5 \\
\hline
\end{tabular}

The results show that variation of the capacitor $C_{L}$ gives rise to frequency shifts and a modification of antenna efficiency, directivity and input impedance. Increasing or decreasing the value of $C_{L}$ yields lower or higher resonant frequencies, efficiency and input impedance, respectively. Directivity remains almost constant and it is always above $2 \mathrm{dBi}$ within the capacitance values under investigation. For the $n=0$ mode the variation of the load inductance $L_{L}$ does not have a strong influence on the properties analyzed. This can be explained by the small current that flows trough these elements (see inset of Fig. 2).

A reconfigurable pattern passive UHF reader loop antenna for RFID applications has also been designed. The antenna parameters are defined in the caption of Fig. 1. However, in this design a loading inductance of $L_{L}=450 \mathrm{nH}$ and a distance between wires $\mathrm{d}=19 \mathrm{~mm}$ have been employed. It has been found through extensive simulations that different mode number, namely $n=0$ and $n=2$ modes, can be obtained at the RFID frequency with two different loading capacitors. Therefore, a passive UHF reader loop antenna with very different properties can be obtained. Fig. 4 shows the simulated return loss characteristic in amplitude with the two selected capacitance values. The resonant frequency of each mode is controlled by the loading capacitors. In particular, the $n=0$ mode was achieved with loading capacitances equal to $0.7 \mathrm{pF}$ as shown by the solid red line. Another value of $30 \mathrm{pF}$ was chosen for the loading capacitors to obtain the same resonance frequency at mode number $n=2$, as shown by the dashed black

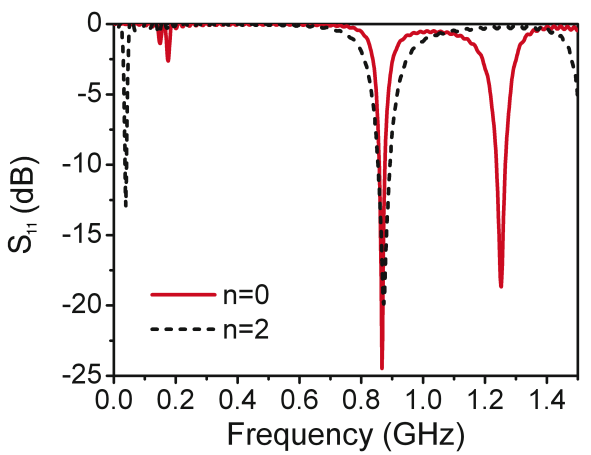

Fig. 4. Return loss of the proposed dual mode reconfigurable passive UHF reader loop antenna working at $868 \mathrm{MHz}$.

line. Fig. 5 shows the farfield radiation pattern obtained for the two operating modes. It can be found that the loop gives an omnidirectional pattern in the $x-y$ plane with a null along the $\mathrm{z}$-axis while working in an $\mathrm{n}=0$ mode. The antenna can switch to a pattern with two main lobes on the z-direction in an $n=2$ mode. A RFID antenna with orthogonal radiation patterns and therefore with almost an isotropic covering area can be achieved.
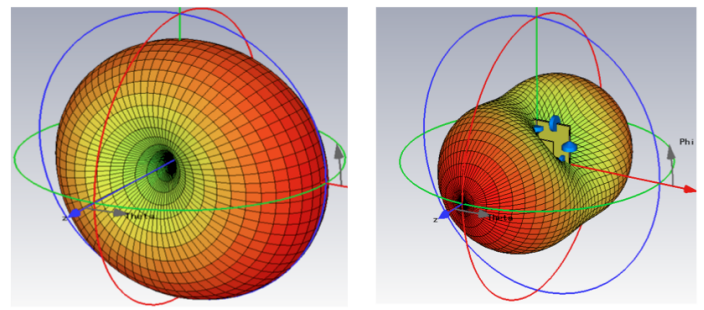

Fig. 5. 3D radiation pattern of the proposed dual mode reconfigurable passive UHF reader loop antenna for $n=0$ and $n=2$ at $868 \mathrm{MHz}$, respectively.

Simulations show that the efficiency and directivity are 84.6 $\%$ and $2.16 \mathrm{dBi}$ for the $\mathrm{n}=0$ mode and $84.3 \%$ and $3.04 \mathrm{dBi}$ for the $\mathrm{n}=2$ mode. It is worth mentioning that in the case of the $n=0$ mode efficiency is bigger compared to that of the previous design.

\section{NEAR-FIELD UHF RFID APPLICATION}

Generally, loop antennas with good magnetic properties have been used as reader antennas in RFID systems. An inductive coupling system is preferred for these applications, since most of the reactive energy is stored in the magnetic field. However, at UHF band the electrical size of the antenna loop becomes comparable to the operating wavelength and it cannot produce an uniform magnetic field. As a result, these antennas produce a weak and nonuniform field distribution at the center of the loop [5]- [7]. In this regard, these problems can be alleviated by metamaterial based antennas working at $\mathrm{n}=0$ mode. For this purpose, the inductively near-field of the loop antenna described in Fig. 1 is analyzed. In this particular case, the distance between the inner and the outer loop is $\mathrm{d}=34 \mathrm{~mm}$ and the load inductance $L_{L}$ is just due to the self-inductance provided by the strip which connects both 
loops. Fig. 6 shows the $y$-axis variation at $\mathrm{z}=0.5 \mathrm{~mm}$ of the magnetic field for the metamaterial loop antenna and a solid loop antenna with the same dimensions.

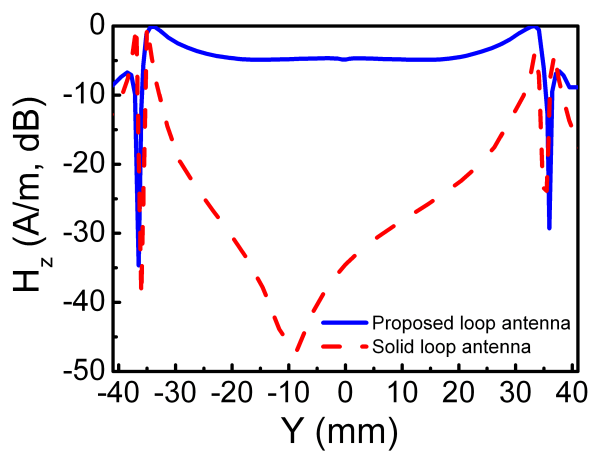

Fig. 6. Simulated magnetic field distribution of the proposed loop antenna in the $y$-axis (rotated $45^{\circ}$ with respect to figure 1) variation. $z=0.5 \mathrm{~mm}$

It is observed that the magnetic field features stronger magnitude in the regions close to the loop lines. Nevertheless, the magnetic field becomes flat along the y-direction in the major portion of the interior zone with low attenuation (minimum value of $-4.7 \mathrm{~dB}$ ). The $\mathrm{x}$-direction presents similar results. On the contrary, the solid-loop antenna suffers a strong H-field attenuation of $-47 \mathrm{~dB}$. The magnitude of the magnetic field close to the center becomes negligible. In order to deeply analyze the performance of the proposed passive UHF near-field reader antenna, a comparative study of different conventional reader antenna configurations, working at the UHF band is carried out. The antennas compared are the proposed loop antenna, a solid loop antenna with the same dimensions of our loop, the loop antenna described in [5], the two segmented loop antennas described in [6] and [7], and the solid loop antenna described in [7]. The dimensions of the antennas presented in [5] - [7] are larger than the proposed loop size. Table II lists the simulated input impedance, directivity, radiation and total efficiency as well as the lowest strength field magnitude achieved.

TABLE II

COMPARATIVE STUDY OF THE NEAR-FIELD UHF LOOP ANTENNA WORKING AT THE N=0 MODE.

\begin{tabular}{|c|c|c|c|c|c|}
\hline Ant. & $\begin{array}{c}\text { Imp. } \\
(\Omega)\end{array}$ & $\begin{array}{c}\text { Dir. } \\
(d B i)\end{array}$ & $\begin{array}{c}E f f_{r} \\
(\%)\end{array}$ & $\begin{array}{c}E f f_{t} \\
(\%)\end{array}$ & $\begin{array}{c}\text { H-field } \\
(A / m, d B)\end{array}$ \\
\hline \hline Loop1 & 50 & 2.5 & 84.3 & 84.1 & -4.7 \\
\hline Loop2 & $66-223 \mathrm{j}$ & 2.59 & 98.7 & 24.9 & -47 \\
\hline$[5]$ & $489-36 \mathrm{j}$ & 4.2 & 94.3 & 32.3 & -26 \\
\hline$[6]$ & $87-5 \mathrm{j}$ & 2.2 & 97.9 & 84 & -25 \\
\hline$[7] .1$ & $146-81 \mathrm{j}$ & 2.5 & 99.1 & 64.2 & -25 \\
\hline$[7] .2$ & $206-57 \mathrm{j}$ & 3.1 & 99 & 77.8 & -42 \\
\hline
\end{tabular}

The left-handed loop exhibits the best input impedance of $50 \Omega$ and the best total efficiency of $84.1 \%$, even if loading elements with higher losses are employed. Matching circuits are required for the rest of the antennas in order to obtain efficiency values higher than $90 \%$. All antennas are low gain. Concerning the near $\mathrm{H}$-field distribution, the proposed loop antenna has the lowest attenuation value. It is at least 100 times lower than the segmented loop antennas and 10000 times lower than the solid loop antennas.

\section{CONCLUSIONS}

In this letter, a novel compact loop antenna for near/far-field RFID applications is presented. The design is based on a transmission line with left-handed loading by means of two series capacitances and a shunt inductance. The antenna has zero phase constant at $868 \mathrm{MHz}$, giving thus an omnidirectional pattern in the plane of the loop. The left-handed loop reader antenna offers improved performances as reconfigurable or optimized responses can easily be achieved. Also, the proposed loop antenna has demonstrated the capability of producing strong magnetic field with relatively uniform distributions in the near-field region of the antenna.This sort of study sheds light onto the potential uses of metamaterials, since conventional and emerging technologies have been compared directly. Several possible applications for these metamaterial based antennas can be foreseen in technologies such as RFID, automotive electronics, TV, and radio reception.

\section{REFERENCES}

[1] K. Finkenzeller, RFID Handbook: Radio- Frequency Identification Fundamentals and Applications, John Wiley and Sons, 2002.

[2] V. D. Hunt, A. Puglia and M. Puglia, RFID - A Guide to Radio Frequency Identification, John Wiley and Sons, Inc, 2007.

[3] K. V. S. Rao, P. V. Nikitin and S. F. Lam, "Antenna Design for UHF RFID Tags: A Review and a Practical Application," IEEE Transactions on Antennas and Propagation, vol. 53, pp. 3870-3876, 2005.

[4] X. Qing and Z. N. Chen, "Proximity effects of metallic environments on high frequency RFID reader antenna: study and applications", IEEE Transactions on Antennas and Propagation, vol. 55, pp. 3105-3111, 2007.

[5] Z. N. Chen, C. K. Goh and X. Qing, "Loop antenna for UHF near-field RFID reader" European Conference on Antennas and Propagation, 12-16 April, 2010.

[6] Y. S. Ong, X. Qing, C. K. Goh and Z. N. Chen, "A Segmented Loop Antenna for UHF Near-Field RFID" Antennas and propagation Society International Symposium, 11-17 July, 2010.

[7] X. Qing, C. K. Goh and Z. N. Chen, "A Broadband UHF Near-Field RFID Antenna" IEEE Transactions on Antennas and Propagation, Vol. 58, pp. 3829-3838, 2010.

[8] A. K. Iyer and G. V. Eleftheriades, "Negative refractive index metamaterials supporting 2-D waves", IEEE MTT-S International Microwave Symposium Digest, vol. 2, pp. 1067-1070, 2002.

[9] C. Caloz and T. Itoh, "Application of the transmission line theory of left- handed (LH) materials to the realization of a microstrip "LH line" ", IEEE-AP-S USNC/URSI National Radio Science Meeting, vol. 2, pp. 412-415, 2002.

[10] A. A. Oliner, "A periodic-structure negative-refractive-index medium without resonant elements", IEEE-AP-S USNC/URSI National Radio Science Meeting, vol. 2, p. 41, 2002.

[11] A. Lai, T. Itoh and C. Caloz, "Composite right/left-handed transmission line meta- materials," IEEE Microwave Magazine, vol. 5, pp. 34-50, 2004

[12] A. L. Borja, J. Kelly, Q. Liu, P. S. Hall and Z. Hu "Dipole antenna with left-handed loading operating at zero order mode", Progress In Electromagnetics Research C, vol. 19, pp. 85-92, 2011.

[13] A. L. Borja, P. Hall, Q. Liu and H. Iizuka, "Omnidirectional loop antenna with left-handed loading", IEEE Antennas and Wireless Propagation Letters, vol. 6, pp. 495-498, 2007.

[14] Q. Liu, P. S. Hall and A. L. Borja, "Efficiency of electrically small dipole antennas loaded with left-handed transmission lines", IEEE Transactions on Antennas and Propagation, vol. 57, pp. 3009-3017, 2009. 